\title{
Preliminary demonstration of benchtop NMR metabolic profiling of feline urine: chronic kidney disease as a case study
}

\author{
Natalie Finch ${ }^{1}$, Benita Percival ${ }^{2}$, Elena Hunter ${ }^{2}$, Robin J. Blagg ${ }^{3}$, Emily Blackwell ${ }^{1}$, James Sagar ${ }^{3}$, \\ Zeeshan Ahmad ${ }^{4}$, Ming-Wei Chang ${ }^{5}$, John A. Hunt ${ }^{2}$, Melissa L. Mather ${ }^{6}$, Séverine Tasker ${ }^{7}$, Luisa De Risio ${ }^{2,7}$ and \\ Philippe B. Wilson ${ }^{2^{*}}$ (D)
}

\begin{abstract}
Objective: The use of benchtop metabolic profiling technology based on nuclear magnetic resonance (NMR) was evaluated in a small cohort of cats with a view to applying this as a viable and rapid metabolic tool to support clinical decision making.

Results: Urinary metabolites were analysed from four subjects consisting of two healthy controls and two chronic kidney disease (CKD) IRIS stage 2 cases. The study identified 15 metabolites in cats with CKD that were different from the controls. Among them were acetate, creatinine, citrate, taurine, glycine, serine and threonine. Benchtop NMR technology is capable of distinguishing between chronic kidney disease case and control samples in a pilot feline cohort based on metabolic profile. We offer perspectives on the further development of this pilot work and the potential of the technology, when combined with sample databases and computational intelligence techniques to offer a clinical decision support tool not only for cases of renal disease but other metabolic conditions in the future.
\end{abstract}

Keywords: Chronic kidney disease, Cat, Metabolite, Metabolomics, NMR

\section{Introduction}

Metabolic profiling involves the investigation of molecules within a biological system and the perturbations within that in response to internal or external stimuli. Metabolic profiling and associated techniques have been previously applied to human, animal, and plant models [1-7]. High-field nuclear magnetic resonance (NMR) and mass spectrometric measurements are considered the gold standard for metabolomic investigations [8-14], however, there are limitations with employing these techniques in point-of-care or near-patient settings, such as veterinary or medical practices, due to the mechanical

\footnotetext{
${ }^{*}$ Correspondence: philippe.wilson@ntu.ac.uk

${ }^{2}$ Nottingham Trent University, Brackenhurst Lane, Southwell NG25 0QF, UK

Full list of author information is available at the end of the article
}

and electrical requirements of such equipment. Benchtop NMR (bNMR) however provides an opportunity for the selectivity of NMR to be utilised and accessible to healthcare and veterinary professionals, providing a wealth of metabolic data to support clinical decision-making. bNMR-based metabolomics has recently shown potential to overcome human health challenges, for example, demonstrating use in point of care settings for human urinary analysis of type 2 diabetic (T2D) patients, with results acquired under 15 min [15-21]. Indeed, bNMR-based metabolic profiling can therefore be proposed as a translational healthcare technology to identify perturbations in metabolites from disease and the environment.

Typically, a multi-platform approach is used to diagnose conditions in felines inclusive of clinical symptoms and physical examination. At present, the diagnosis of felines for some elements of renal diseases are dependent 
on histology and are invasive procedures, requiring tissue biopsies [11]. However, cognitive biases are present upon observing tissues, and this is not a quantitative method of diagnosis due to the discipline predominantly being qualitative or semi-qualitative [11]. Alternative methods include the growth of aerobic bacteria using aerobic urine culture, which is time-consuming and has potential contamination risks [12]. Moreover, abdominal radiographs and ultrasounds can be used to support clinical examinations however these require owner intervention and physical intervention of the clinician at the surgery [12]. Furthermore, particularly over the COVID-19 pandemic period, veterinary consultations took place remotely either via teleconference or video link [19]. Diagnostic tests routinely performed are inherently invasive, and particularly complicated in a period of lockdown to contain a global pandemic, as well as being particularly difficult based on behavioural factors in some domesticated species; however, collecting material for urinary analysis is relatively facile and benchtop NMR, once clinically validated, offers the potential for highly rigorous remote investigations [21-25]. Practically, for such remote collections to be of value to the community, simple post-sampling steps can be implemented by owners; in a time where it is now commonplace to selfadminister lateral flow tests, the post-collection stabilisation of the urine sample prior to transfer to a laboratory would only involve a sterilisation phase which could be simply carried out through addition of a pre-aliquoted solution (containing reagents described in Experimental section) to the collected urine sample. Therefore, to detect chronic kidney disease (CKD) in the early stages, changes in metabolites in urine (as a proximal sample) can be identified. Such analysis can provide a deeper understanding of the mechanisms and metabolic pathways key to the progression of CKD. Cats with CKD are staged according to guidelines developed by the International Renal Interest Society (IRIS) and accepted by the American and European Societies of Veterinary Nephrology and Urology. The IRIS stages range from no azotemia (IRIS stage 1) to the most severe azotemia (IRIS stage 4). Staging guidelines are helpful for making diagnostic, prognostic and therapeutic recommendations for CKD.

\section{Main text}

Free-catch, fasted urine samples were collected from cats clinically diagnosed with CKD from urinalysis, GFR and serum biochemistry assessments and centrifuged immediately (3500 rpm at $4{ }^{\circ} \mathrm{C}$ for $15 \mathrm{~min}$ ). Healthy subjects were recruited from the local institutional cat population and had no clinically diagnosed underlying health issues. All subjects were administered similar diets. The method of urine collection was identical to case subjects above. A minimum of $0.5 \mathrm{ml}$ of urine is required for analysis. The supernatants were then stored at $-80{ }^{\circ} \mathrm{C}$ prior to analysis. NMR spectra were obtained on an Oxford Instruments X-Pulse $60 \mathrm{MHz}$ benchtop NMR spectrometer, operating at $+40{ }^{\circ} \mathrm{C}$. Samples were defrosted, and diluted by addition of $20 \%$ (by volume) deuterium oxide, $\mathrm{D}_{2} \mathrm{O}$. One-dimensional proton NMR spectra (with and without solvent suppression), and proton-proton gradient-selective COSY spectra were obtained for each sample. One-dimensional ${ }^{1} \mathrm{H}$ spectra were collected with 64 scans, $6 \mathrm{~s}$ acquisition time and $5 \mathrm{~s}$ relaxation delay; one-dimensional solvent suppressed ${ }^{1} \mathrm{H}$ spectra [using a WET (Water suppression Enhanced through $T_{1}$ effects) sequence] were collected with 128 scans, and the same acquisition time, and relaxation delay; COSY spectra were obtained with 8 scans of 256 slices. All spectra were internally referenced to $\mathrm{H}_{2} \mathrm{O} / \mathrm{HOD}$ at $\delta_{\mathrm{H}}+4.66 \mathrm{ppm}$. The Kyoto Encyclopedia of Gene and Genomics (KEGG) was used to ascribe significant biomolecular modifications and describe linkages between metabolic cycles.

High-field metabolomics has already demonstrated translational capability for diagnostic and therapeutic aims in humans with renal conditions [26]. Herein, we use CKD as a case study for the application of this technology and present pilot data from four subjects consisting of two control (Subjects S2,S4) and two with CKD IRIS stage 2 (S1, S3). Subjects S2 and S4 were clinically assessed by a veterinarian for confounding conditions and assessment as control participants for this pilot study as healthy controls with no renal conditions and serum creatinine concentrations of $<145 \mu \mathrm{mol} / \mathrm{L}$. Further details on participants availbale within data protection regulations is available in the Additional file 1.

Subjects S1 and S3 were diagnosed with azotaemic CKD at Stage 2 according to IRIS guidelines from their serum creatinine concentrations of 193 and $188 \mu \mathrm{mol} / \mathrm{L}$, respectively. Additionally, these CKD subjects show stronger resonances in the aromatic region (signal 17) ascribed to hippurate and phenylacetylglycine aromatic protons. Increases in urinary creatinine have previously been confirmed in cats with CKD [5]. Creatine is essential for energy transfer to skeletal muscle through the formation of ATP. Renal dysfunction can lead to an increase in creatinine in urine; therefore, the level of creatinine in urine is a principal indicator of CKD.

Furthermore, the relatively weak acetate signal compared to healthy controls is indicative of decreased excretion in individuals with CKD, as displayed in Fig. 1 (S1, S3). Indeed, an inverse correlation between urinary excretion of acetate and renal function has been established in comparative physiological studies [4]. It has been observed elsewhere that the level of acetate was lower in humans with diabetes mellitus and 


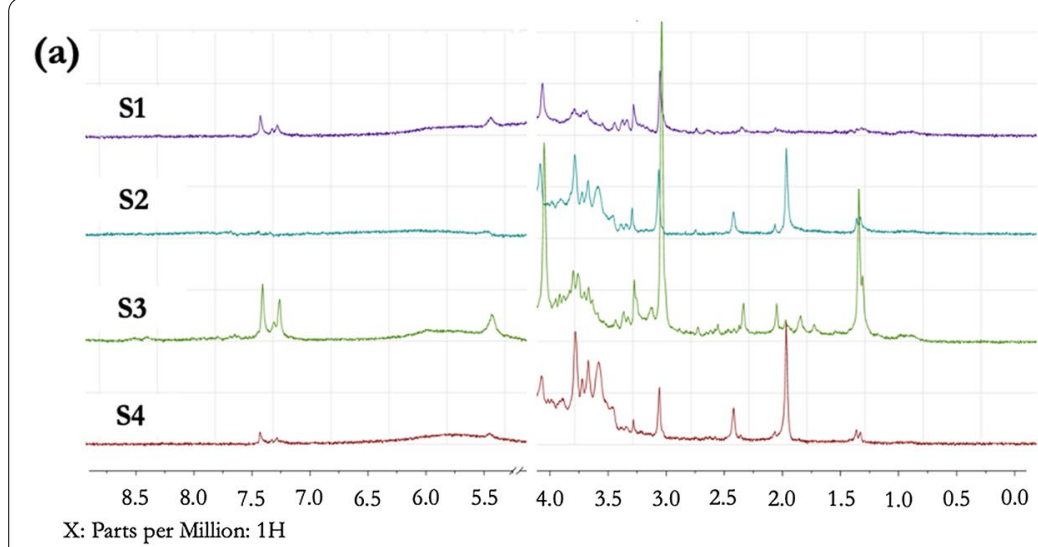

(b)

(c)
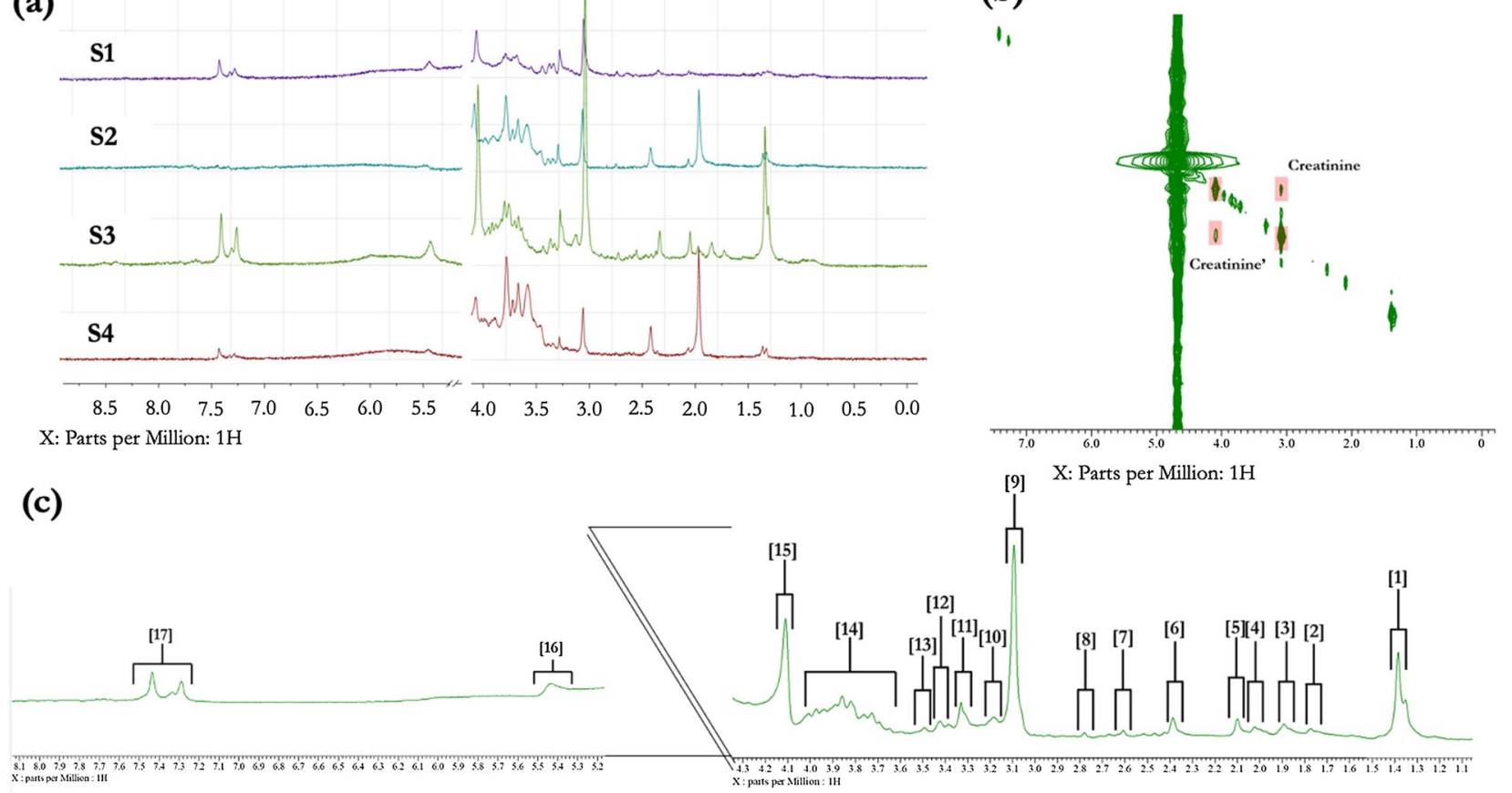

Fig. 1 a urinary NMR metabolic profiles from feline subjects S1-S4 collected at $60 \mathrm{MHz}$ operating frequency; b 2-dimensional COSY spectrum of signal confirmation for S3 sample showing creatinine cross-peaks; c assigned regions of S3 urinary profile with the following assignments: [1] 3-Hydroxybutyrate/Lactate- $\mathrm{CH} 3 /$ Felinine- $\mathrm{CH} 3$ [2] Tentative Felinine Derivative-CH3 [3] Tentative Felinine-CH2 [4] Acetate-CH3 [5] N-Acetyl [6] Pyruvate- $\mathrm{CH} 3$ [7] Citrate- $\mathrm{CH} 2 \mathrm{AB}$ [8] Citrate- $\mathrm{CH} 2 \mathrm{AB}$ [9] Creatinine/Creatine- $\mathrm{N}-\mathrm{CH} 2$ [10] Felinine- $\mathrm{CH} 2$ [1 1] TMAO-N-CH3/Taurine- $\mathrm{CH} 2 / \mathrm{Betaine}-\mathrm{CH} 3$ [12] Taurine- $\mathrm{CH} 2$ [13] Glycine- $\mathrm{CH} 2$ [14] Felinine- $\mathrm{CH} 2$ [15] Creatinine- $\mathrm{CH} 2$ [16] Tentative Allantoin and Urea-NH2 [17] Aromatic signals consisting of Hippurate-CHs and phenylacetylglycine- $\mathrm{CHs}$

CKD than those with CKD alone [13]. Reduced excretion of acetate in urine indicates further metabolism to acetyl coenzyme A which has a central role in fatty acid metabolism. Acetyl coenzyme A is involved in the central carbon metabolism that subsequently generates ATP through catabolism of the acetyl moiety in the tricarboxylic acid cycle [27]. Indeed, a reduction in urinary excretion of TCA cycle metabolites and renal expression of the genes which regulate these metabolites has been demonstrated in human cases of CKD, linking to mitochondrial dysfunction and CKD progression. Moreover, recent genomic and metabolomic assessments of human patients with non-diabetic CKD identified reduced TCA cycle activity in cases when compared to a control group. This reduction in urinary excretion of TCA cycle metabolites was linked to a reduction in overall mitochondrial biogenesis in kidney tissues from CKD patients likely caused by reduced expression of genes such as isocitrate dehydrogenase 3 in the tubointerstitial compartment of the kidney [9].

Metabolic pathway analysis (MPA) from these pilot data identified that glycine, serine and threonine metabolism was associated with classification between CKD and control subjects. An impact score from pathway topological analysis was 0.3 , whilst a $p$ value adjusted by the Holm-Bonferroni correction was 0.00426 . This suggests that glycine, serine and threonine metabolism are modified in CKD subjects compared to the controls. Concentration decreases in metabolites excreted through both serine and threonine metabolism were detected elsewhere $[10,28]$. Such a significance of serine metabolism between groups could be an indicator of its biological role in renal dysfunction. Serine acts as a mediator for methylation and the lowering of blood pressure in renal mechanisms. Serine excretion is correlated to glomerular filtration rate (GFR) which in-turn is used to define reductions in renal function. Therefore, if GFR is reduced, $D$-serine begins to accumulate in tissue $[10,15]$.

Furthermore, changes in glycine concentration in biofluids over the course of CKD has identified perturbations in amino acid metabolism in both rat and human models [18]. Additionally, hippuric acid metabolite signals in CKD subjects are linked to glycine conjugation with benzoic acid in hepatic, intestinal and renal activity [22]. Taken together, these glycine and glycine-conjugated metabolites are linked to oxidative stress and 
inflammation through both the I $\mathrm{KB} \alpha / \mathrm{NF}-\kappa \mathrm{B}$ and Keap1/ Nrf2 pathways [23]. Moreover, glycine forms a central node in glutathione metabolism, which with tocopherol act as baseline markers of oxidation and concentrations of downstream metabolites are highly mediated by CKD stage. Cellular processes determining immunity, but particularly inflammation can be assessed by sphingolipid metabolites where sphingosines act as signalling molecules. These metabolites, also detected as N-acetyl functions (signal 5) have been demonstrated to be highly sensitive in their concentration to dietary interventions for the treatment of CKD in cats. Indeed, successful fibre supplementation aligned with positive clinical outcomes to changes in diet were ascribed to increased sphingolipid metabolite concentrations in plasma of cats with CKD [8].

Regarding other metabolites, the reduction of urine citrate concentration has also been associated with CKD where urine citrate can prevent the formation of calciumbased kidney stones [17]. Furthermore, the dysfunction of taurine was connected to CKD in other studies [2]. Taurine is involved in osmoregulation, calcium ion kinetics and regulation of the membrane potential in skeletal muscle. Moreover, taurine can be considered as anti-inflammatory and an antioxidant agent [25]. Kidneys have a crucial role in maintaining the level of taurine. However, the levels of taurine can be dramatically decreased in patients with CKD. Therefore, taurine can also feature one of the main regulatory metabolites for the detection of CKD [2].

Renal function can be assessed by the measurement of glomerular filtration rate and is often referred to as the gold standard, however this can involve clinically and technically challenging measurements. Indeed, CKD can be diagnosed in small animals through a combinatorial approach involving creatinine concentration and urine specific gravity, and whilst these may be widely used, they remain insensitive as prognostic and monitoring markers. Our preliminary assessment of a pilot feline cohort identifies (a) the ability of low field NMR spectroscopy to detect $>15$ metabolites in feline urine and (b) the potential of the technology when applied to large cohorts and informed by machine learning, to provide fast biofluid analysis to support clinical decision making.

Benchtop NMR metabolic profiling offers an opportunity to leverage the selectivity of NMR spectroscopy in a portable format capable of being more widely applied, whilst taking advantage of chemometric methods to deconvolute spectra and offer the facility as a technique with diagnostic potential. Benchtop NMR is therefore a potential tool for the early detection of diseases and the evaluation of health conditions that can provide necessary, timely treatments $[29,30]$.

\section{Limitations}

Whilst the authors are fully cognisant that the small sample sizes in this preliminary and pilot exemplar study do not allow for the substantiated development of a rationale for use in biomarker discovery or elucidation, we intend this article to act as a first case study or case report in the application of this miniaturised technology within the field of animal medicine. Indeed, further to our work in human medicine [20], a number of more specific feline metabolites (such as felinine) also need to be analytically quantified in terms of instrumental sensitivity in order to allow us to fully establish working thresholds for the technology in terms of limits of detection and quantification.

\section{Supplementary Information}

The online version contains supplementary material available at https://doi. org/10.1186/s13104-021-05888-y.

Additional file 1: Table S1. Available data on study participants.

Table S2. Labelled metabolites identified in urine from subjects S1-S4 and shown in manuscript Figure 1 (a)-(c)

\begin{abstract}
Acknowledgements
Not applicable

Authors' contributions

NF, BP, EH, RJB, EB, JS, ZA, MC, JH, MM, ST, LDR, and PBW. All authors contributed to the study conception and design. Material preparation was performed by NF, EB, RJB, BP, EH and JS. Data collection and analysis were performed by $R J B, J S, B P, E H, Z A$ and MC. Supervision was performed by PBW, ZA, MM, JH, ST and LDR. The first draft of the manuscript was written by PBW, MM, ST, LDR and $\mathrm{J}$ and all authors commented on previous versions of the manuscript. All authors have read and approved the final manuscript.
\end{abstract}

\section{Funding}

PBW thanks Oxford Instruments (OI) for their research support and colleagues for collaborating in the running of this study as detailed in "Authors' Contributions" section. OI colleagues Drs James Sagar and Robin Blagg supported: material preparation and data collection and analysis. Further details on individual contributions to the study are in the "Authors' Contributions" section.

\section{Availability of data and materials}

The datasets used and/or analysed during the current study available from the corresponding author on reasonable request.

\section{Code availability}

Not applicable.

\section{Declarations}

Ethics approval and consent to participate

All procedures involving animals were in compliance with the European Community Council Directive of 24 November 1986. Written informed consent was obtained from the owners and the study was conducted with approval from the University Animal Welfare and Ethics Review Board (UIN UB/12/025).

Consent for publication

Not applicable.

Competing interests

Robin J. Blagg and James Sagar are employed by Oxford Instruments. 


\section{Author details}

${ }^{1}$ University of Bristol Veterinary School, Langford House, Langford, Bristol BS40 5DU, UK. ${ }^{2}$ Nottingham Trent University, Brackenhurst Lane, Southwell NG25 OQF, UK. ${ }^{3}$ Oxford Instruments Magnetic Resonance, Tubney Woods, Abingdon, Oxfordshire OX13 5QX, UK. ${ }^{4}$ De Montfort University, The Gateway, Leicester LE1 9BH, UK. ${ }^{5}$ Nanotechnology and Integrated Bioengineering Centre, University of Ulster, Jordanstown Campus, Newtownabbey, Northern Ireland, UK. ${ }^{6}$ University of Nottingham, University Park, Nottingham NG7 2RD, UK. ${ }^{7}$ Linnaeus Veterinary Limited, Friars Gate, Solihull B90 4BN, UK.

Received: 20 October 2021 Accepted: 14 December 2021

Published online: 24 December 2021

\section{References}

1. Basoglu A, Baspinar N, Tenori L, Licari C, Gulersoy E. Nuclear magnetic resonance (NMR)-based metabolome profile evaluation in dairy cows with and without displaced abomasum. Vet Q. 2020;40:1-15. https://doi. org/10.1080/01652176.2019.1707907.

2. Chen DQ, Cao G, Chen H. Identification of serum metabolites associating with chronic kidney disease progression and anti-fibrotic effect of 5-methoxytryptophan. Nat Commun. 2019;10:1-9. https://doi.org/10. 1038/s41467-019-09329-0.

3. Cesaremarincola F, Mannina L. Special issue on "NMR-based metaboIomics and its applications volume 2." Metabolites. 2020;10:45. https://doi. org/10.3390/metabo10020045.

4. Davies R. The metabolomic quest for a biomarker in chronic kidney disease. CKJ. 2018;11:694-703. https://doi.org/10.1093/ckj/sfy037.

5. Ferlizza E, Campos A, Neagu A, Cuoghi A, Bellei E, Monari E, Dondi F, Almeida AM, Isani G. The effect of chronic kidney disease on the urine proteome in the domestic cat (Felis catus). Vet J. 2015;204(1):73-81.

6. Giraldi M, Paltrinieri SC, Scarpa P. Serum concentration of homocysteine in spontaneous feline chronic kidney disease. Vet J. 2019. https://doi.org/ 10.1016/j.tvjl.2019.105358.

7. Gottlieb S, Rand J. Managing feline diabetes: current perspectives. Vet Med. 2018;9:33-42. https://doi.org/10.2147/VMRR.S125619.

8. Hall JA, Jackson MI, Jewell DE, Ephraim E. Chronic kidney disease in cats alters response of the plasma metabolome and fecal microbiome to dietary fiber. PloS ONE. 2020;15(7): e0235480.

9. Hallan S, Afkarian M, Zelnick LR, Kestenbaum B, Sharma S, Saito R, Darshi M, Barding G, Raftery D, Ju W, Kretzler M. Metabolomics and gene expression analysis reveal down-regulation of the citric acid (TCA) cycle in non-diabetic CKD patients. EBioMedicine. 2017;26:68-77.

10. Hesaka A, Sakai S, Hamase K, Ikeda T, Matsui R, Mita M, Horio M, Isaka Y, Kimura T. D-Serine reflects kidney function and diseases. Sci Rep. 2019;25(9):5104. https://doi.org/10.1038/s41598-019-41608-0.

11. Jones $E$, et al. Predicting diagnosis of Australian canine and feline urinary bladder disease based on histologic features. Vet Sci. 2020;7:190. https:// doi.org/10.3390/vetsci7040190.

12. Kaul E, Hartmann K, Reese S, Dorsch R. Recurrence rate and long-term course of cats with feline lower urinary tract disease. J Feline Med Surg. 2020;22:544-56. https://doi.org/10.1177/1098612X19862887.

13. Kim DK, Kim Y. SAT-198 Significant urinary metabolites in the progression of chronic kidney disease. Kidney Int. 2019;4:590.

14. Kim HS, Kim ET, Eom JS, Choi YY, Lee SJ, et al. Exploration of metabolite profiles in the biofluids of dairy cows by proton nuclear magnetic resonance analysis. PLoS ONE. 2021;16: e0246290. https://doi.org/10.1371/ journal.pone.0246290.

15. Kimura T, Hesaka A, Isaka Y. D-Amino acids and kidney diseases. Clin Exp Nephrol. 2020;24:404-10. https://doi.org/10.1007/s10157-020-01862-3.

16. Leenders J, Grootveld M, Percival B, Gibson M, Casanova F, Wilson PB. Benchtop low-frequency $60 \mathrm{MHz}$ NMR analysis of urine: a comparative metabolomics investigation. Metabolites. 2020;10:155. https://doi.org/10. 3390/metabo10040155.

17. Liu J-J, Liu S, Gurung RL, Ching J, Kovalik JP, Tan TY, Lim SC. Urine tricarboxylic acid cycle metabolites predict progressive chronic kidney disease in type 2 diabetes. J Clin Endocrinol Metab. 2018;103:4357-64. https://doi. org/10.1210/jc.2018-00947.

18. Mahbub MH, Yamaguchi N, Takahashi H, et al. Relationship of reduced glomerular filtration rate with alterations in plasma free amino acids and uric acid evaluated in healthy control and hypertensive subjects. Sci Rep. 2019;9:10252. https://doi.org/10.1038/s41598-019-46598-7.

19. Pearson C. Coping with covid-19. Vet Rec. 2020;188:422.

20. Percival BC. Low-field, benchtop NMR spectroscopy as a potential tool for point-of-care diagnostics of metabolic conditions: validation, protocols and computational models. High Throughput. 2018. https://doi.org/10. 3390/ht8010002.

21. Percival BC, Gibson M, Leenders J, Wilson PB, Grootveld M. Univariate and multivariate statistical approaches to the analysis and interpretation of NMR-based metabolomics datasets of increasing complexity. In: Wilson PB, Grootveld M, editors. Computational techniques for analytical chemistry and bioanalysis. Cambridge: Royal Society of Chemistry Theoretical Chemistry Series; 2020. p. 1-40.

22. Poon K, Pang KS. Benzoic acid glycine conjugation in the isolated perfused rat kidney. Drug Metab Dispos. 1995;23:255-60.

23. Sivandzade F, Prasad S, Bhalerao A, Cucullo L. NRF2 and NF-қB interplay in cerebrovascular and neurodegenerative disorders: Molecular mechanisms and possible therapeutic approaches. Redox Biol. 2019;21: 101059. https://doi.org/10.1016/j.redox.2018.11.017.

24. Sun $H$, Wang B, Wang J, et al. Biomarker and pathway analyses of urine metabolomics in dairy cows when corn stover replaces alfalfa hay. J Anim Sci Biotechnol. 2016;7:49. https://doi.org/10.1186/s40104-016-0107-7.

25. Trachtman H, Sturman JA. Taurine: a therapeutic agent in experimental kidney disease. Amino Acids. 1996;11:1-13. https://doi.org/10.1007/ BF00805717.

26. Tugnoli V, et al. H-NMR and 13C-NMR lipid profiles of human renal tissues. Biopolymers. 2003;72:86-95. https://doi.org/10.1002/bip.10299.

27. Walsh CT, Tu BP, Yang Y. Eight kinetically stable but thermodynamically activated molecules that power cell metabolism. Chem Rev. 2018;118:1460-94. https://doi.org/10.1021/acs.chemrev.7b00510.

28. Wang YN, Ma SX, Chen YY, Chen L, Liu BL, Liu QQ, Zhao YY. Chronic kidney disease: biomarker diagnosis to therapeutic targets. Clin Chim Acta. 2019;499:54-63. https://doi.org/10.1016/j.cca.2019.08.030.

29. Zhang J, Wei S, Liu L, Nagana GA, Bonney P, Stewart J, Knapp DW. NMRbased metabolomics study of canine bladder cancer. Biochim Biophys Acta. 2012;1822:1807-14. https://doi.org/10.1016/j.bbadis.2012.08.001.

30. Zhang G, Rupasri M, Wishart DS, Ametaj BN. A multi-platform metabolomics approach identifies urinary metabolite signatures that differentiate ketotic from healthy dairy cows. Front vet sci. 2021;8:8. https://doi. org/10.3389/fvets.2021.595983.

\section{Publisher's Note}

Springer Nature remains neutral with regard to jurisdictional claims in published maps and institutional affiliations.

Ready to submit your research? Choose BMC and benefit from:

- fast, convenient online submission

- thorough peer review by experienced researchers in your field

- rapid publication on acceptance

- support for research data, including large and complex data types

- gold Open Access which fosters wider collaboration and increased citations

- maximum visibility for your research: over 100M website views per year

At BMC, research is always in progress.

Learn more biomedcentral.com/submissions 